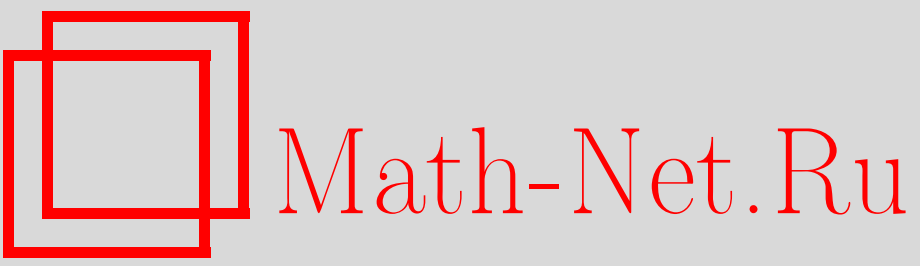

С. В. Белютин, Исследование точной интегрируемости многоволнового нелинейного уравнения Шредингера, ТМФ, 1997, том 110, номер 2, 242-253

DOI: https://doi.org/10.4213/tmf965

Использование Общероссийского математического портала Math-Net.Ru подразумевает, что вы прочитали и согласны с пользовательским соглашением

http://www . mathnet.ru/rus/agreement

Параметры загрузки:

IP: 44.207 .124 .84

26 апреля 2023 г., 15:36:20 
ТЕОРЕТИЧЕСКАЯ

И МАТЕМАТИЧЕСКАЯ

ФИЗИКА

Том 110, № 2

февраль, 1997

С.В. Белютин ${ }^{*}$

\section{ИССЛЕДОВАНИЕ ТОЧНОЙ ИНТЕГРИРУЕМОСТИ МНОГОВОЛНОВОГО НЕЛИНЕЙНОГО УРАВНЕНИЯ ШРЕДИНГЕРА}

Доказано, что многоволновое нелинейное уравнение Шредингера, описывающее эволюцию нескольких почти монохроматических волн с равными групповыми скоростями, не является точно интегрируемым (в смысле существования бесконечной последовательности локальных законов сохранения и симметрий). Исследована точная интегрируемость систем вида $w_{t}^{i}=\alpha_{i} w_{x x}^{i}+a_{k l m}^{i} w^{k} w^{l} w^{m}$ с невырожденной диагональной матрицей при старшей производной.

\section{1. ВВЕДЕНИЕ}

Хорошо известно, что распространение почти монохроматическойплоской волны малой конечной амплитуды в среде с пренебрежимо малой диссипацией и сильной дисперсией описывается нелинейным уравнением Шредингера (НУШ) $[1,2]$

$$
\begin{gathered}
i A_{t}+\alpha A_{x x}+\beta A|A|^{2}=0, \\
\varphi=A e^{\theta}+\text { c.c. }, \quad \theta=i(k x-\omega t), \quad \alpha=\frac{1}{2} \frac{\partial^{2} \omega}{\partial k^{2}},
\end{gathered}
$$

где $\omega$ и $k$ обозначают частоту и волновое число, $\varphi$ - возмушение некоторого равновесного состояния системы. НУШ относится к числу знаменитых уравнений, и его свойства достаточно подробно изучены. Поэтому только коротко изложим суть явления, описываемого данным уравнением. Гармонические возмушения при распространении в среде с сильной дисперсией претерпевают слабое изменение длины волны $l$ и соответственно волнового числа $k=2 \pi / l$, прямо пропорциональное амплитуде возмушения - именно этот физический факт лежит в основе универсальности НУШ. Если обозначить через $\varepsilon$ амплитуду волны, обезразмеренную по некоторому характерному параметру задачи ( $\varepsilon$ отвечает за нелинейность и характеризует отклонение системы от равновесного состояния), то подобное изменение будет соответствовать возникновению волн (так называемых боковых гармоник) с волновыми числами $k$ такими, что $\left(k-k_{0}\right) / k_{0} \sim \varepsilon$. Боковые гармоники, взаимодействуя с основной волной и между собой, порождают на временах порядка $\left(\varepsilon \omega_{0}\right)^{-1}$ возмушения нулевой (в гидродинамике называемое индуцированным

${ }^{*}$ Институт механики Московского государственного университета 


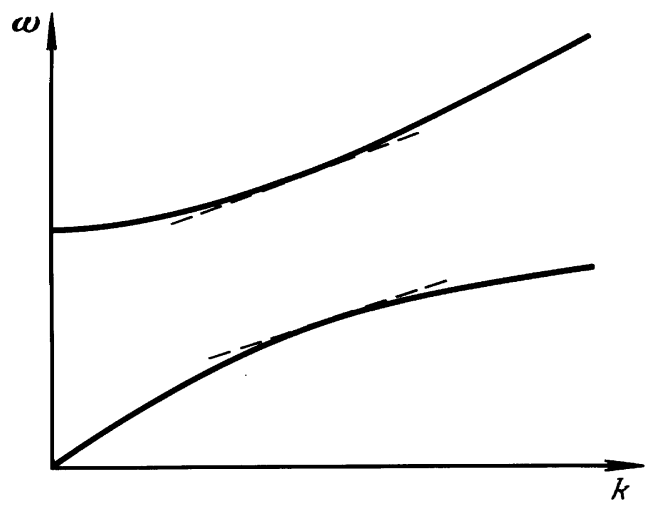

Рис. 1. Характерный вид дисперсионного соотношения для волн в плазме: верхняя ветвь ленгмюровская, нижняя - ионноакустическая.

средним течением) и второй гармоник. Эти возмущения, в свою очередь, на временах порядка $\left(\varepsilon^{2} \omega_{0}\right)^{-1}$ начинают влиять на несущую волну и вызывают медленное изменение ее амплитуды, что и описьвается НУШ. Таким образом, НУШ описывает модуляцию или демодуляцию почти гармонической волны под влиянием нелинейного самовоздействия волны и дисперсионных свойств среды (подчеркнем, что речь при этом идет только о линейно устойчивых волнах).

К числу удивительных свойств НУШ, помимо его точной интегрируемости, относится также то, что оно согласуется с экспериментальными данными гораздо лучше, чем следовало бы ожидать исходя из тех предположений, при которых оно выводилось [3].

В заключение вводной части, касающейся непосредственно НУШ, стоит отметить, что требование о совпадении невозмушенного состояния $\varphi=0$ именно с равновесным не является строго обязательным: в [4], например, говорится, что НУШ (а точнее, уравнение Ландау-Гинзбурга - НУШ с добавленной малой диссипацией) позволяет описывать возникновение когерентных структур из состояния пространственного хаоса, отличаюшегося от термодинамического равновесия.

Известно также, что совместное распространение нескольких почти монохроматических волн (все прочие условия остаются теми же, что и для НУШ) описывается системой уравнений $[5,6]$

$$
\begin{gathered}
i \frac{\partial A^{m}}{\partial t}+\alpha_{m} \frac{\partial^{2} A^{m}}{\partial x^{2}}+A^{m} \sum_{l=1}^{N} \gamma_{m l}\left|A^{l}\right|^{2}=0 \\
\alpha_{m}=\frac{1}{2} \frac{d^{2} \omega}{d k^{2}} \quad\left(k=k_{m}\right), \quad m=1, \ldots, N .
\end{gathered}
$$

Здесь следует отметить, что координата $x$ во всех уравнениях системы одинакова только в том случае, если все волны имеют одну и ту же групповую скорость $C_{\mathrm{g}} \equiv \frac{d \omega}{d k}$ $\left(k=k_{j}\right)[7]$. Фактически система $(1.2)$, как и НУШ, представляет собой запись в системе координат, движушейся с групповой скоростью. Если же условие равенства групповых скоростей не выполняется, то нужно вводить новую независимую переменную и вид системы значительно усложняется. 


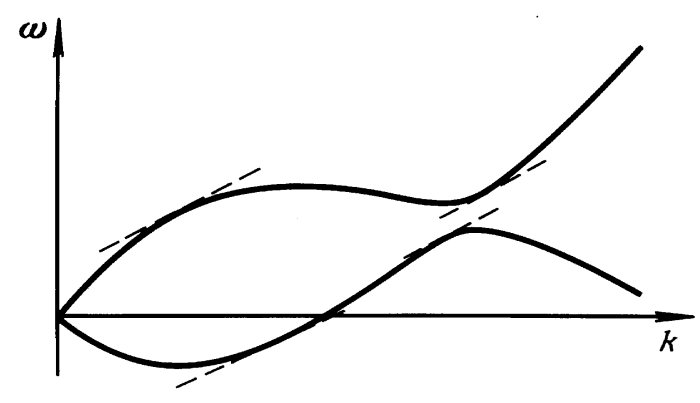

Рис. 2. Характерный вид дисперсионного соотношения для гравитационно-капиллярных волн в двухслойной жидкости (в некотором диапазоне параметров).

Волны с равными групповыми скоростями существуют во многих средах. Условие равенства групповых скоростей соответствует точкам с параллельными касательными на графике дисперсионного соотношения $\omega(k)$. Особенно просто это условие выполняется, если дисперсионное соотношение для данной среды имеет более одной ветви (см. рис. 1, 2).

В дальнейшем систему (1.2) будем называть системой равных групповых скоростей (РГС). Система РГС, как и НУШ, описывает дисперсию и нелинейное самовоздействие волн, а также дополнительно учитывает нелинейное взаимодействие между волнами, что выражается в присутствии нелинейных перекрестных членов.

Если рассмотреть эволюцию нескольких почти монохроматических волн с одинаковыми дисперсионными и нелинейными свойствами, т.е. с одинаковыми $k_{j}, \omega\left(k_{j}\right), \omega^{\prime}\left(k_{j}\right)$ и $\omega^{\prime \prime}\left(k_{j}\right)$ (практически это соответствует возможности как-то выделить или пометить несколько составляющих одной и той же волны, характерный пример - волны света с различной поляризацией в нелинейной оптике), то она будет описываться векторным НУШ

$$
i \frac{\partial A^{m}}{\partial t}+\alpha \frac{\partial^{2} A^{m}}{\partial x^{2}}+\beta A^{m} \sum_{l=1}^{N}\left|A^{l}\right|^{2}=0 .
$$

Векторное НУШ является частным случаем системы (1.2), для которой требуется только совпадение групповых скоростей $\omega^{\prime}\left(k_{j}\right)$.

Свойства НУШ и векторного НУШ достаточно хорошо изучены, так как эти уравнения интегрируемы методом обратной задачи рассеяния [8-10]. Интересно, что и некоторые другие близкородственные системы уравнений, как, например, уравнения длинно-коротковолнового и трехволнового резонансов, также являются точно интегрируемыми. Поэтому закономерно возникает вопрос о точной интегрируемости системы (1.2). Данная статья и будет посвящена решению этого вопроса.

\section{2. ИССЛЕДОВАНИЕ ТОЧНОЙ ИНТЕГРИРУЕМОСТИ}

Будем изучать интегрируемость системы уравнений с произвольной кубической нелинейностью

$$
\begin{gathered}
w_{t}^{i}=\alpha_{i} w_{x x}^{i}+a_{k l m}^{i} w^{k} w^{l} w^{m}, \quad i, k, l, m=1, \ldots, N \\
\alpha_{i} \neq 0, \quad \alpha_{i} \neq \alpha_{j}, \quad \forall i, j, \quad i \neq j
\end{gathered}
$$


(здесь и далее используется немое суммирование по всем повторяюшимся индексам, кроме $i$ и $j$ ), и упорядочим систему так, чтобы выполнялось

$$
\alpha_{i}=-\alpha_{2 M-i+1} \quad \text { при } \quad i=1, \ldots, 2 M, \quad 0 \leq 2 M \leq N .
$$

Будем также считать (для краткости формулировок утверждений), что система не содержит линейных уравнений.

Элементы тензора $a$ определяются по системе уравнений неоднозначно, поэтому потребуем, чтобы выполнялось соотношение

$$
a_{k l m}^{i}=a_{l k m}^{i}=a_{m k l}^{i}
$$

Систему (2.1) можно записать также в векторном виде:

$$
\mathbf{w}_{t}=\Lambda \mathbf{w}_{x x}+\frac{1}{3} \Phi \mathbf{w}, \quad \Phi_{j}^{i}=3 a_{l m j}^{i} w^{l} w^{m}
$$

здесь $\Lambda$ - диагональная матрица с коэффициентами $\alpha_{i}$ вдоль главной диагонали.

Система РГС (1.2) для $M$ волн приводится к виду $(2.1),(2.2)$ заменами

$$
t \rightarrow i t, \quad A^{m}=w^{m}, \quad \overline{A^{m}}=w^{2 M-m+1} .
$$

Нелинейное уравнение Шредингера (1.1) также представимо в виде (2.1):

$$
\begin{aligned}
& w_{t}^{1}=\alpha w_{x x}^{1}+\beta\left(w^{1}\right)^{2} w^{2}, \\
& w_{t}^{2}=-\alpha w_{x x}^{2}-\beta\left(w^{2}\right)^{2} w^{1}
\end{aligned}
$$

Такие пары уравнений иногда будем для краткости называть НУШ.

Введем некоторые новые обозначения и ряд определений, следуя работам [11-13]. Прежде всего обозначим

$$
w_{k}^{j} \equiv \frac{\partial^{k} w^{j}}{\partial x^{k}}
$$

и будем считать $w_{k}^{j}$ новыми независимыми переменными.

ОПРЕДЕЛЕНИЕ 1. Система уравнений

$$
\mathbf{u}_{t}=\mathbf{F}\left(\mathbf{u}, \mathbf{u}_{1}, \mathbf{u}_{2}, \ldots, \mathbf{u}_{m}\right)
$$

имеет локальный закон сохранения, если сушествует функция $\rho\left(\mathbf{u}, \mathbf{u}_{1}, \ldots, \mathbf{u}_{n}\right)$, называемая сохраняющейся плотностью (или для краткости плотностью), такая что

$$
\frac{d \rho}{d t}=\mathfrak{D} \sigma, \quad \mathfrak{D} \equiv \frac{d}{d x}
$$


ОпредЕлЕниЕ 2. Операция линеаризации (производная Фреше) сопоставляет вектору $\mathbf{S}\left(\mathbf{u}, \mathbf{u}_{1}, \ldots, \mathbf{u}_{m}\right)$ дифференциальный оператор

$$
\mathbf{S}_{*}=\sum_{k=0}^{m} \frac{\partial \mathbf{S}}{\partial \mathbf{u}_{k}} \cdot \mathfrak{D}^{k}
$$

Для системы $(2.1) F_{*}=\Lambda \mathfrak{D}^{2}+\Phi$.

ОПРЕДЕЛЕНИЕ 3 . Порядком $n$ сохраняюшейся плотности $\rho_{n}$ (и соответствующего закона сохранения) называется порядок дифференциального оператора

$$
\mathbf{R}=\left(\frac{\delta \rho}{\delta \mathbf{u}}\right)_{*} .
$$

Плотность называется невырожденной, если матрица при старшем порядке оператора $\mathfrak{D}^{n}$ является невырожденной.

В качестве определения точной интегрируемости выбирается существование у системы (2.1) бесконечной последовательности невырожденных локальных законов сохранения сколь угодно большого порядка.

Теперь можно сформулировать следуюшую лемму.

ЛЕмма. Система (2.1) имеет невырожденный локальный закон сохранения порядка $n \geq 3$ тогда и только тогда, когда она распадается на пары уравнений (2.5), әквивалентные НУШІ.

ДокАЗАТЕЛЬСТво. Пусть система (2.1) имеет локальный закон сохранения порядка $n$. Взяв вариационную производную $\delta / \delta w^{j}$ и производную Фреше от $(2.8)$, получим эквивалентную операторную форму закона сохранения:

$$
\partial_{t} \mathbf{R}+\mathbf{R}\left(\Lambda \mathfrak{D}^{2}+\Phi\right)+\left(\Lambda^{T} \mathfrak{D}^{2}+\Phi^{T}\right) \mathbf{R}+\mathbf{P}\left(\Phi^{T}\right)_{*}=0
$$

где обозначено

$$
\begin{gathered}
\mathbf{P}=\left(P_{1}, \ldots, P_{N}\right)^{T}, \quad P_{j}=\delta \rho / \delta w^{j} \\
\mathbf{R}=(\mathbf{P})_{*}, \quad \mathbf{R}=\sum_{k=0}^{n} R_{k} \mathfrak{D}^{k}, \quad\left(R_{k}\right)_{i j}=\frac{\partial P_{i}}{\partial w_{k}^{j}}
\end{gathered}
$$

верхний индекс $T$ означает транспонирование матрищы.

Разложим уравнение (2.11) по степеням оператора $\mathfrak{D}$ :

$$
\begin{gathered}
\sum_{k=0}^{n}\left[\partial_{t}\left(R_{k}\right) \mathfrak{D}^{k}+R_{k} \Lambda \mathfrak{D}^{k+2}+R_{k} \sum_{r=0}^{k} C_{k}^{r} \mathfrak{D}^{k-r}(\Phi) \mathfrak{D}^{r}+\Lambda R_{k} \mathfrak{D}^{k+2}+\right. \\
\left.+2 \Lambda \mathfrak{D}\left(R_{k}\right) \mathfrak{D}^{k+1}+\Lambda \mathfrak{D}^{2}\left(R_{k}\right) \mathfrak{D}^{k}+\Phi^{T} R_{k} \mathfrak{D}^{k}\right]+\mathbf{P} \cdot\left(\Phi^{T}\right)_{*}=0 \\
C_{k}^{r}=\frac{k !}{r !(k-r) !}
\end{gathered}
$$


и введем по аналогии с коммутатором двух матриц новое обозначение

$$
\langle A, B\rangle=A B+B^{T} A,
$$

где $A$ и $B-$ произвольные квадратные матрицы. Тогда коэффициент при $\mathfrak{D}^{n+2}$ равен

$$
\left\langle R_{n}, \Lambda\right\rangle=0
$$

следовательно, только элементы $\left(R_{n}\right)_{i, 2} M-i+1$ могут быть отличны от нуля (здесь учтено упорядочение (2.2)).

В порядке $\mathfrak{D}^{n+1}$ выполняется

$$
\left\langle R_{n-1}, \Lambda\right\rangle+2 \Lambda^{T} \mathfrak{D}\left(R_{n}\right)=0
$$

следовательно, $R_{n}=$ const и только $\left(R_{n-1}\right)_{i, 2 M-i+1} \neq 0$ из всех элементов матрицы $R_{n-1}$.

Обозначим $R_{n} \equiv K$. Из полученных выше условий следует, что $\rho_{n}$ эквивалентно (совпадает с точностью до полной производной)

$$
\rho_{n} \simeq \sum_{i} K_{i, 2 M-i+1} w_{n}^{i} w^{2 M-i+1}+\cdots, \quad i=1, \ldots, M
$$

где точками обозначены слагаемые, имеющие меньший порядок производных. Из определения $K$ следует (а из (2.16) это очевидно), что должно выполняться соотношение

$$
K_{2 M-i+1, i}=(-1)^{n} K_{i, 2 M-i+1}
$$

Невырожденность плотности $\rho_{n}$ эквивалентна по определению невырожденности матрицы $K$. Таким образом, можно считать $K_{i, 2 M-i+1} \neq 0$ и $N=2 M$.

Система (2.1) однородна относительно замены $t^{\prime}=\lambda^{2} t, x^{\prime}=\lambda x,\left(w^{i}\right)^{\prime}=\lambda w^{i}$. Отсюда и из (2.16) следует, что можно рассматривать только полиномиальные однородные сохраняющиеся плотности. В этом случае коэффициенты $R_{k}$ будут однородными полиномами веса $(n-k)$ и возникающие в дальнейшем константы интегрирования можно считать равными нулю.

В порядке $\mathfrak{D}^{n}$ получаем

$$
2 \Lambda^{T} \mathfrak{D}\left(R_{n-1}\right)=-\left(\left\langle R_{n-2}, \Lambda\right\rangle+\langle K, \Lambda\rangle\right)
$$

Это уравнение разрешимо только в случае, если в правой части элементы с индексами $i, 2 M-i+1$ равны нулю, т.е.

$$
K_{i, 2 M-i+1} \Phi_{2 M-i+1}^{2 M-i+1}+\Phi_{i}^{i} K_{i, 2 M-i+1}=0 \Rightarrow \Phi_{2 M-i+1}^{2 M-i+1}=-\Phi_{i}^{i}
$$

здесь суммирования по $i$ нет. Константы интегрирования выбираем нулевыми, поэтому $R_{n-1}=0$. 
Рассмотрим сначала элементы матриц $\left(R_{k}\right)_{i j}, k=0, \ldots, n-2$, с индексами $j \neq$ $2 M-i+1$. Для квадратичных по $w$ частей этих матриц можно получить точные формулы:

$$
\begin{gathered}
\left(R_{n-k}^{Q}\right)_{i j}=\left(R_{n-k}^{\mathrm{I}}\right)_{i j}+\left(R_{n-k}^{\mathrm{II}}\right)_{i j}, \\
\left(R_{n-k}^{\mathrm{I}}\right)_{i j}=\sum_{r=1}^{p}(-1)^{k-r} C_{k-r-1}^{r-1} \frac{\left(2 \alpha_{i}\right)^{k-2 r}}{\left(\alpha_{i}+\alpha_{j}\right)^{k-r}} \mathfrak{P}_{i}^{r-1} \mathfrak{D}^{k-2 r} W_{i j}^{\mathrm{I}}, \\
\left(R_{n-k}^{\mathrm{II}}\right)_{i j}=\sum_{r=0}^{q-1} \sum_{m=1}^{k-2 r-2}(-1)^{k-r-m-1} C_{k-r-m-2}^{r} c_{n}^{m} \times \\
\quad \times \frac{\left(2 \alpha_{i}\right)^{k-2 r-m-2}}{\left(\alpha_{i}+\alpha_{j}\right)^{k-r-m-1}} \mathfrak{P}_{i}^{r} \mathfrak{D}^{k-2 r-2} W_{i j}^{\mathrm{II}}, \\
W_{i j}^{\mathrm{I}}=K_{i, 2 M-i+1} \Phi_{j}^{2 M-i+1}+\Phi_{i}^{2 M-j+1} K_{2 M-j+1, j}, \\
W_{i j}^{\mathrm{II}}=K_{i, 2 M-i+1} \Phi_{j}^{2 M-i+1}, \quad\left(R_{n-2}^{\mathrm{II}}\right)_{i j}=0, \quad \mathfrak{P}_{i}=\partial_{t}+\alpha_{i} \mathfrak{D}^{2}, \\
k=2, \ldots, n, \quad p=[k / 2], \quad q=[(k-1) / 2], \quad j \neq 2 M-i+1,
\end{gathered}
$$

верхний индекс $Q$ означает квадратичную часть.

Условие, которое получается в порядке $\mathfrak{D}^{1}$, эквивалентно формальному условию $\left(R_{-1}^{Q}\right)_{i j}=0$, где $\left(R_{-1}^{Q}\right)_{i j}$ определяется согласно $(2.20)$ при $k=n+1$. Рассмотрим сначала случай четного $n, n=2 p$. Тогда $\left(R_{-1}^{Q}\right)_{i j}$ можно представить в виде

$$
\left(R_{-1}^{Q}\right)_{i j}=\sum_{k=1}^{p} \mathfrak{D}^{2 k-1}\left(\phi_{2 k-1}\right)_{i j}
$$

Из условия $\mathfrak{D}^{1}\left(\phi_{1}\right)_{i j}=0$ получаем

$$
\left(\alpha_{l}+\alpha_{m}\right) \alpha_{j} K_{i, 2 M-i+1} a_{l m j}^{2 M-i+1}=\left(\alpha_{l}+\alpha_{m}\right) \alpha_{i} K_{j, 2 M-j+1} a_{l m i}^{2 M-j+1}
$$

а из условия $\mathfrak{D}^{3}\left(\phi_{3}\right)_{i j}=0-$

$$
\left(\alpha_{l}+\alpha_{m}\right)\left(\alpha_{i}-\alpha_{j}\right) K_{i, 2 M-i+1} a_{l m j}^{2 M-i+1}=0 .
$$

Теперь рассмотрим элементы с индексами $i, 2 M-i+1$. Из условия в порядке $\mathfrak{D}^{n-1}$ находим

$$
\left(R_{n-2}\right)_{i, 2 M-i+1}=-\frac{n}{2 \alpha_{i}} K_{i, 2 M-i+1} \Phi_{2 M-i+1}^{2 M-i+1}
$$

а в порядке $\mathfrak{D}^{n-2}$ получится уравнение

$$
\begin{aligned}
\mathfrak{D}\left(R_{n-3}\right)_{i, 2 M-i+1}= & \frac{n}{\left(2 \alpha_{i}\right)^{2}} K_{i, 2 M-i+1}\left[-\left(\alpha_{l}+\alpha_{m}\right) a_{l, m, 2 M-i+1}^{2 M-i+1} w_{1}^{l} w_{1}^{m}+\right. \\
& \left.+a_{l, m, 2 M-i+1}^{2 M-i+1} \mathfrak{D}\left(\alpha_{l} w_{1}^{l} w^{m}+\alpha_{m} w^{l} w_{1}^{m}\right)\right]+\mathfrak{D}^{2}\left(\phi_{n-3}\right)_{i, 2 M-i+1}
\end{aligned}
$$


которое имеет решение, только если

$$
\left(\alpha_{l}+\alpha_{m}\right) K_{i, 2 M-i+1} a_{l, m, 2 M-i+1}^{2 M-i+1}=0 .
$$

Анализируя условия (2.21) и (2.23), убеждаемся, что ненулевыми могут быть только элементы вида $a_{i, 2}^{i}-i+1, i$ (с точностью до перестановок нижних индексов) и $a_{i i i}^{2 M-i+1}$. Чтобы разобраться с элементами вида $a_{i i i}^{2 M-i+1}$, обратимся к условию для порядка $\mathfrak{D}^{0}$ :

$$
\partial_{t}\left(R_{0}^{Q}\right)_{i j}+\left(\mathbf{P} \cdot\left(\Phi^{T}\right)_{*}\right)_{i j}^{Q}+\mathfrak{D}^{n}(K \Phi)_{i j}=0
$$

где

$$
\begin{aligned}
\left(\mathbf{P} \cdot\left(\Phi^{T}\right)_{*}\right)_{i j}^{Q}= & 6 K_{2 M-i+1, i} a_{i i i}^{2 M-i+1} w_{n}^{i} w^{i}+ \\
& +6 K_{i, 2 M-i+1} a_{i, 2 M-i+1, i}^{i} w_{n}^{2 M-i+1} w^{2 M-i+1}
\end{aligned}
$$

При $n=2 p$ условие (2.24) можно записать в виде

$$
\sum_{k=0}^{p} \mathfrak{D}^{2 k}\left(\psi_{2 k}\right)_{i j}=0
$$

Приравнивая нулю $\mathfrak{D}^{0}\left(\psi_{0}\right)_{i j}$ и $\mathfrak{D}^{2}\left(\psi_{2}\right)_{i j}$, находим, что $a_{i i i}^{2 M-i+1}=0$.

Итак, остались только элементы $a_{i, 2 M-i+1, i}^{i}$. С учетом (2.19) имеем

$$
a_{i, 2 M-i+1, i}^{i}=-a_{2 M-i+1, i, 2 M-i+1}^{2 M-i+1},
$$

и каждая пара уравнений для $w^{i}$ и $w^{2 M-i+1}$ эквивалентна НУШ.

Случай нечетного $n, n=2 p+1$, рассматривается аналогично.

Теперь рассмотрим систему вида (2.1) с произвольной диагональной невырожденной матрищей $\Lambda$, т.е. допустим совпадаюшие коэффициенты $\alpha_{i}=\alpha_{j} \neq 0$. Но прежде чем формулировать основную теорему, напомним, что точная интегрируемость систем уравнений

$$
\begin{array}{rlrl}
u_{t}^{i} & =u_{x x}^{i}+a_{k l m}^{i} u^{k} v^{l} u^{m}, & & i=1, \ldots, N, \\
v_{t}^{i}=-v_{x x}^{i}-\tilde{a}_{k l m}^{i} v^{k} u^{l} v^{m}, & i=1, \ldots, M,
\end{array}
$$

была исследована в работах $[11,14]$. Интегрируемые системы вида $(2.25)$ будем называть обобщенными НУШ.

Teopema. Сucmema

$$
w_{t}^{i}=\alpha_{i} w_{x x}^{i}+a_{k l m}^{i} w^{k} w^{l} w^{m}, \quad \alpha_{i} \neq 0, \quad i, k, l, m=1, \ldots, N,
$$

имеет невырожденный локальный закон сохранения порядка $n \geq 3$ тогда и только тогда, когда она распадается на обобщенные НУШІ. 
ДокАЗАТЕЛЬСТво проводится аналогично доказательству леммы, но с использованием блочной структуры матрищ; в частности, элементы с индексами $R_{i, 2} M-i+1$ заменяются матричными блоками $R_{i j}$ с индексами $i, j$ такими, что $\alpha_{i}+\alpha_{j}=0$. В результате в случае закона сохранения четного порядка условия (2.21) и (2.23) заменяются на условие

$$
\left(\alpha_{l}+\alpha_{m}\right)\left(\alpha_{i}-\alpha_{j}\right) \sum_{s, \alpha_{s}=-\alpha_{i}} K_{i s} a_{l m j}^{s}=0,
$$

суммы по $l, m, i$ и $j$ нет.

Пусть $\alpha_{1}, \ldots, \alpha_{k}$ - множество всех различных коэффициентов $\alpha_{i}$ системы (2.26). Обозначим через $\mathfrak{N}\left(\alpha_{i}\right)$ множество индексов $s$ таких, что $\alpha_{s}=\alpha_{i}$. Введем матрицы $K_{(i)}$, $i=1, \ldots, k$, составленные из элементов $K_{s m}, s \in \mathfrak{N}\left(\alpha_{i}\right), m \in \mathfrak{N}\left(-\alpha_{i}\right)$. Нетрудно убедиться, что невырожденность большой матрицы $K$ эквивалентна тому, что все матрицы $K_{(i)}$ являются квадратными (т.е. $\left.\operatorname{card} \mathfrak{N}\left(-\alpha_{i}\right)=\operatorname{card} \mathfrak{N}\left(\alpha_{i}\right)\right)$ и невырожденными. Поэтому если индекс $i$ пробегает все значения из $\mathfrak{N}\left(\alpha_{i}\right)$, то $(2.27)$ при $\left(\alpha_{l}+\alpha_{m}\right) \times$ $\left(\alpha_{i}-\alpha_{j}\right) \neq 0$ представляет собой умножение невырожденной матрицы на вектор. Перебирая все возможные комбинации индексов, находим, что ненулевыми элементами тензора $a$ с верхним индексом $i$ могут быть только те $a_{k l m}^{i}$, у которых $k, m \in \mathfrak{N}\left(\alpha_{i}\right)$, $l \in \mathfrak{N}\left(-\alpha_{i}\right)$ или $k, l, m \in \mathfrak{N}\left(-\alpha_{i}\right)$ (система распалась на подсистемы с равными по модулю $\left.\alpha_{i}\right)$. Анализируя условие в порядке $\mathfrak{D}^{0}(2.24)$, убеждаемся, что $a_{k l m}^{i}=0$ при $k, l, m \in \mathfrak{N}\left(-\alpha_{i}\right)$ и система распадается на обобшенные НУШ.

Случай закона сохранения нечетного порядка рассматривается аналогично.

ОПРЕДЕЛЕНИЕ 4. Система уравнений (2.7) имеет симметрию $\mathbf{u}(\tau, t, x)$,

$$
\mathbf{u}_{\tau}=\mathbf{G}\left(\mathbf{u}, \mathbf{u}_{1}, \ldots, \mathbf{u}_{k}\right)
$$

если выполняется

$$
\partial_{t} \mathbf{G}=\partial_{\tau} \mathbf{F}
$$

Порядком симметрии называется порядок дифференциального оператора $\mathbf{G}_{*}$.

ЗАмЕчАниЕ. Теорема, сформулированная для законов сохранения, справедлива и для симметрий.

ДокАЗАТЕЛьСтво. Возьмем производную Фреше от (2.28) и получим эквивалентную операторную формулировку определения симметрии

$$
\partial_{t} \mathbf{G}_{*}-\partial_{\tau} \mathbf{F}_{*}+\left[\mathbf{G}_{*}, \mathbf{F}_{*}\right]=0 \text {. }
$$

Дальнейшие выкладки проводятся аналогично выкладкам для законов сохранения.

\section{3. ПРИЧИНЫ НЕИНТЕГРИРУЕМОСТИ}

В связи с отсутствием точной интегрируемости системы (1.2) следует обратить внимание на одно обстоятельство. Уравнения РГС, как и многие другие эволюционные амплитудные уравнения, можно получить с помошью метода многих масштабов. В то же время хорошо известно, что между точной интегрируемостью и многомасштабными разложениями сушествует тесная связь $[15,16]$. В работе [16] было сформулировано 
утверждение о сохранении свойств $C$-интегрируемости (сведение к линейному уравнению с помощью замены переменных) и $S$-интегрируемости (интегрируемость с помощью спектральных преобразований) при многомасштабных разложениях; т.е., применив такое разложение к точно интегрируемому уравнению (системе уравнений), мы должны получить амплитудное уравнение (или систему уравнений), также являющееся точно интегрируемым.

Полученный выше результат находится в кажушемся противоречии с данным утверждением. Однако это противоречие легко устранимо. Дело в том, что в $[15,16]$ рассматривались только многомасштабные разложения, учитьвающие не более одной “дисперсии”. Это значит, что при изучении эволюции системы волн дисперсионные эффекты либо не учитьвались вовсе, что соответствует сохранению только первого члена в зависимости частоты волны от расплывания волнового пакета

$$
\omega-\omega_{0}=C_{\mathrm{g}}\left(k-k_{0}\right)+\frac{1}{2} \frac{d^{2} \omega}{d k^{2}}\left(k-k_{0}\right)^{2}+o\left(\left(k-k_{0}\right)^{3}\right)
$$

где $k_{0}$ - волновое число исходного пакета, либо учитывались для одной волны, либо учитывались только для волн, имеюших одинаковые $C_{\mathrm{g}}=d \omega / d k$ и $d^{2} \omega / d k^{2}$. Поясним это на наиболее известных примерах. Одноволновые уравнения, такие как КдФ, НУШ и др., учитывают, естественно, только одну дисперсию. Уравнения трехволнового резонанса не учитывают дисперсионные эффекты (данный пример показывает, что с несколькими различными групповыми скоростями интегрируемость уживается). При длинно-коротковолновом резонансе дисперсия учитывается только для короткой волны. Наконец, в векторных КдФ и НУШ дисперсионные свойства всех волн принимаются одинаковыми.

Именно для таких многомасштабных разложений (учитываюших не более одной дисперсии) и справедливо утверждение о сохранении точной интегрируемости. Однако, как следует из предыдушего раздела, учет более сложных дисперсионных эффектов приводит к потере этого свойства, может “портить" интегрируемость. Поэтому в формулировке утверждения, приведенного в работе [16], следует добавить слова “учитывающих не более одной дисперсии".

Теперь необходимо только убедиться на каком-либо конкретном примере, что интегрируемость действительно может нарушаться, поскольку возможна ситуация, когда из неинтегрируемого уравнения получается неинтегрируемое, а из интегрируемого при применении той же самой процедуры многомасштабного разложения получается интегрируемое за счет, например, обрашения в нуль некоторых коэффициентов (подобные случаи рассматривались в [16]).

В качестве примера выберем точно интегрируемое уравнение Буссинеска:

$$
u_{t t}-\left(a u_{x x x x}+b\left(u^{2}\right)_{x x}+c u_{x x}\right)=0 .
$$

Рассмотрим простейший случай только двух взаимодействуюших волн (обозначен- 
ных ниже индексами “+” и “-”). Разлагая стандартным образом:

$$
\begin{aligned}
u & =\sum_{k=1}^{\infty} \varepsilon^{k} u_{k}, \quad \partial_{t}=\sum_{k=0}^{\infty} \varepsilon^{k} \partial / \partial t_{k}, \quad \partial_{x}=\sum_{k=0}^{\infty} \varepsilon^{k} \partial / \partial x_{k}, \\
u_{1} & =A_{+} \exp \theta_{+}+A_{-} \exp \theta_{-}+\text {c.c. }, \\
\theta_{ \pm} & =i\left(k_{ \pm} x_{0}-\omega_{ \pm} t_{0}\right), \quad A_{ \pm}=A_{ \pm}\left(X, t_{2}, x_{2}, \ldots\right), \\
X & =x_{1}+C_{\mathrm{g}} t_{1}, \quad \frac{\partial}{\partial T}=\frac{\partial}{\partial t_{2}}+C_{\mathrm{g}} \frac{\partial}{\partial x_{2}}
\end{aligned}
$$

и при условии

$$
\omega_{+}^{\prime}\left(k_{+}\right)=\omega_{-}^{\prime}\left(k_{-}\right) \equiv C_{\mathrm{g}}
$$

получим в третьем приближении по $\varepsilon$ систему уравнений РГС для двух волн

$$
\begin{aligned}
& i \frac{\partial A_{+}}{\partial T}+\frac{1}{2} \omega_{+}^{\prime \prime}\left(k_{+}\right) \frac{\partial^{2} A_{+}}{\partial X^{2}}+\left(\beta_{+}\left|A_{+}\right|^{2}+\gamma_{+}\left|A_{-}\right|^{2}\right) A_{+}=0 \\
& i \frac{\partial A_{-}}{\partial T}+\frac{1}{2} \omega_{-}^{\prime \prime}\left(k_{-}\right) \frac{\partial^{2} A_{-}}{\partial X^{2}}+\left(\beta_{-}\left|A_{-}\right|^{2}+\gamma_{-}\left|A_{+}\right|^{2}\right) A_{-}=0
\end{aligned}
$$

где

$$
\begin{gathered}
\beta_{+}=-\frac{b k_{+}^{2}}{\omega_{+}} \frac{b \cdot\left(2 k_{+}\right)^{2}}{P\left(2 k_{+}, 2 \omega_{+}\right)}, \\
\gamma_{+}=-\frac{b k_{+}^{2}}{\omega_{+}}\left[\frac{b \cdot\left(k_{+}+k_{-}\right)^{2}}{P\left(k_{+}+k_{-}, \omega_{+}+\omega_{-}\right)}+\frac{b \cdot\left(k_{+}-k_{-}\right)^{2}}{P\left(k_{+}-k_{-}, \omega_{+}-\omega_{-}\right)}\right] \\
\omega_{+}^{\prime \prime}=\left(c-6 a k_{+}^{2}-C_{\mathrm{g}}^{2}\right) / \omega_{+}, \quad C_{\mathrm{g}}=\frac{k_{ \pm}\left(c-2 a k_{ \pm}^{2}\right)}{\omega_{ \pm}}
\end{gathered}
$$

а $\beta_{-}, \gamma_{-}, \omega_{-}^{\prime \prime}$ получаются перестановкой “+” и “-”; через $P(k, \omega)$ обозначено дисперсионное соотношение:

$$
P(k, \omega) \equiv \omega^{2}+a k^{4}-c k^{2}=0 .
$$

Выберем некоторые $k_{+}$и $k_{-}$, удовлетворяющие условию (3.2), например:

$$
k_{+}=\sqrt{\frac{c}{4 a}}, \quad k_{-}=\sqrt{\frac{2 c}{3 a}}, \quad \omega_{+}=\frac{\sqrt{3 a}}{4} \frac{c}{a}, \quad \omega_{-}=-\frac{\sqrt{2 a}}{3} \frac{c}{a} .
$$

Тогда

$$
\begin{aligned}
\omega_{+}^{\prime \prime}\left(k_{+}\right) & =-10 \sqrt{3 a} / 9, \quad \omega_{-}^{\prime \prime}\left(k_{-}\right)=5 \sqrt{2 a}, \\
\gamma_{+} & =-\frac{3}{2} \frac{b k_{+}^{2}}{c \omega_{+}} \neq 0, \quad \gamma_{-}=-\frac{3}{2} \frac{b k_{-}^{2}}{c \omega_{-}} \neq 0,
\end{aligned}
$$

т.е. система не является обобщенным НУШ $\left(\left|\omega_{+}^{\prime \prime}\right| \neq\left|\omega_{-}^{\prime \prime}\right|\right)$ и не распадается на независимые НУШ ( $\gamma_{+}$и $\gamma_{-}$не равны нулю), поэтому она не интегрируема.

Таким образом, наличие двух различных дисперсий действительно может “портить" интегрируемость уравнения.

Автор выражает благодарность академику Р.И. Нигматулину, Н.А. Гумерову и С.И. Свинолупову за поддержку и внимание к работе. 


\section{Список литературы}

[1] Р. Додд, Дж. Эйлбек, Дж. Гиббон, Х. Моррис. Солитоны и нелинейные волновые уравнения. М.: Мир, 1988.

[2] A. Ньюәлл. Солитоны в математике и физике. М.: Мир, 1989.

[3] H. C. Yuen, B. M. Lake. Adv. Appl. Mech. 1982. V. 22. P. 67-229.

[4] A. С. Монин. О когерентных структурах в турбулентных течениях. В кн.: Этюды о турбулентности. М.: Наука, 1994. С. 8-16.

[5] D. J. Benney, A. C. Newell. J. Math. and Phys. 1967. V. 46. P. 133-139

[6] D. J. Benney, G. J. Roskes. Studies Appl. Math. 1969. V. 48. № 4. P. 377-385.

[7] С. В. Белютин. Модуляционная неустойчивость волн и резонанс групповых скоростей в двухслойной жидкости. Дисс. канд. физ.-матем. наук. Тюмень: ТГУ, 1993.

[8] B. Е. Захаров, А. Б. Шабат. ЖЭТФ. 1971. Т. 61. С. 118-134.

[9] В. Е. Захаров, С. В. Манаков. ТМФ. 1974. Т. 19. № 3. С. 332-343.

[10] С. В. Манаков. ЖЭТФ. 1973. Т. 65. Вып. 2. С. 505-516.

[11] S. I. Svinolupov. Commun. Math. Phys. 1992. V. 143. P. 559-575.

[12] А.В. Михайлов, А.Б. Шабат, В. В. Соколов. Симметрийный подход к классификации интегрируемых уравнений. В кн.: Интегрируемость и кинетические уравнения для солитонов. Киев: Наукова думка, 1990.

[13] А. В. Михайлов, А. Б. Шабат. ТМФ. 1985. Т. 62. № 2. С. 163-185.

[14] A. Fordy, P. Kulish. Commun. Math. Phys. 1983. V. 89. P. 427-443.

[15] V. E. Zakharov, E. A. Kuznetsov. Physica D. 1986. V. 18. P. 455-463.

[16] $\Phi$. Калоджеро. Почему некоторые уравнения одновременно широко распространены и интегрируемы?. В кн.: Интегрируемость и кинетические уравнения для солитонов. Киев: Наукова думка, 1990.

Поступила в редакцию $10 . \mathrm{IV} .1995$ г., после доработки 5.VIII.1996 г.

\section{S. V. Belyutin \\ INVESTIGATION OF THE EXACT INTEGRABILITY OF THE MULTIWAVE SCHRÖDINGER EQUATION}

It is shown that the multiwave nonlinear Schrödinger equation, which describes evolution of several quasi-monochromatic waves with equal group velocities, is not exactly integrable: it does not have infinite sequence of local conservation laws and symmetries. Exact integrability of the system of equations $w_{t}^{i}=\alpha_{i} w_{x x}^{i}+a_{k l m}^{i} w^{k} w^{l} w^{m}$ with the nondegenerate diagonal matrix at the second-order derivative is studied. 